\title{
Erratum: Analysis of environmental isotopes in groundwater to understand the response of a vulnerable coastal aquifer to pumping: Western Port Basin, south-eastern Australia
}

\author{
Matthew Currell • Dioni I. Cendón • Xiang Cheng
}

Erratum to: Hydrogeology Journal

DOI 10.1007/s10040-013-1017-9

A monitoring well, bore ID 71856 , is indicated as being screened in the Sherwood formation in Tables 1 and 2; in fact it is screened within the Older Volcanics unit. This error was noticed after the proofs were approved for publication.

The corrected Tables 1 and 2 are reproduced below.

Published online: 19 September 2013

(C) Springer-Verlag Berlin Heidelberg 2013

The online version of the original article can be found at http://dx.doi.org/ 10.1007/s10040-013-1017-9.

M. Currell (曲)

School of Civil, Environmental and Chemical Engineering,

RMIT University, GPO Box 2476, Melbourne, VIC 3001, Australia e-mail: matthew.currell@rmit.edu.au

Tel.: +61-3-99250402

D. I. Cendón

Australian Nuclear Science and Technology Organisation,

Locked Bag 2001, Kirrawee, NSW 2232, Australia

D. I. Cendón

School of Biological and Environmental Sciences,

The University of New South Wales, Sydney, NSW 2052, Australia

X. Cheng

Department of Primary Industries, Parkville, VIC 3010, Australia 


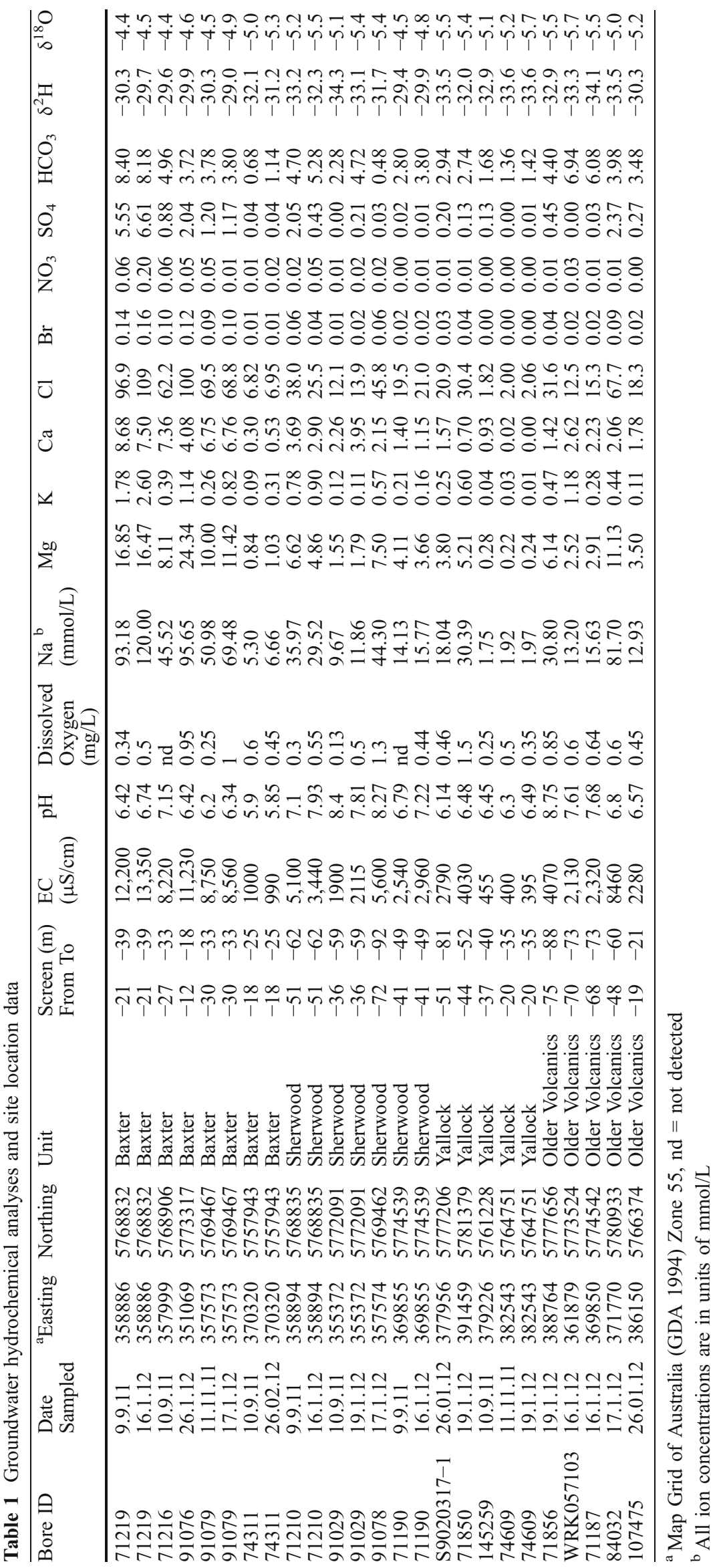


Table 2 Radioisotope data from groundwater

\begin{tabular}{|c|c|c|c|c|c|c|c|c|c|}
\hline $\begin{array}{l}\text { Sample } \\
\text { code }^{\mathrm{a}}\end{array}$ & Bore $\mathrm{ID}^{\mathrm{b}}$ & $\begin{array}{l}\text { Distance } \\
\text { from coast } \\
(\mathrm{km})\end{array}$ & $\begin{array}{l}\text { Geological } \\
\text { Unit }\end{array}$ & $\begin{array}{l}\text { Depth } \\
\text { (screen } \\
\text { midpoint) }\end{array}$ & $\begin{array}{l}\text { Tritium } \\
(\mathrm{TU})^{\mathrm{c}}\end{array}$ & $\begin{array}{l}{ }^{14} \mathrm{C} \\
(\mathrm{pMC})\end{array}$ & $\begin{array}{l}\delta^{13} \mathrm{C} \\
(\% 0)\end{array}$ & $\begin{array}{l}\text { Age } \\
\text { (Uncorrected) }\end{array}$ & $\begin{array}{l}\text { Calcite } \\
\text { saturation } \\
\text { index }\end{array}$ \\
\hline OZO861 & 71219 & 0.5 & Baxter & -30 & 0.03 & 23.91 & -18.4 & 11490 & -0.14 \\
\hline OZO856 & 91076 & 4.5 & Baxter & -15 & 0.23 & 73.17 & -16.3 & 2510 & -1.04 \\
\hline OZO865 & 91029 & 2.9 & Sherwood & -47.5 & 0.02 & 0.91 & -12.4 & 37790 & 0.75 \\
\hline OZO862 & 71210 & 0.5 & Sherwood & -56.5 & 0.01 & 3.09 & -17.2 & 27930 & -0.65 \\
\hline OZO859 & 71190 & 7.8 & Sherwood & -45 & 0.02 & 21.09 & -10.6 & 12500 & -0.46 \\
\hline OZO864 & 91078 & 0.5 & Sherwood & -77 & 0.02 & 24.95 & 18.2 & 11,150 & -0.19 \\
\hline OZO866 & S9020317-1 & 14.2 & Yallock & -66 & 0.08 & 6.59 & -18.2 & 21850 & -1.55 \\
\hline OZO869 & 71850 & 23.7 & Yallock & -48 & 0.01 & 21.77 & -19 & 12250 & -1.64 \\
\hline OZO858 & 74311 & 0.05 & Yallock & -21 & 0.04 & 48.53 & -17.8 & 5810 & -2.58 \\
\hline OZO868 & 74609 & 14.1 & Yallock & -27.5 & 0.1 & 61.15 & -15.5 & 3950 & -3.02 \\
\hline OZO867 & 71856 & 23.5 & Older Volcanics & -81.5 & 0.01 & 4.99 & -21.9 & 24080 & 1.03 \\
\hline OZO870 & WRK057103 & 5.2 & Older Volcanics & -71.5 & 0.05 & 1.39 & -17.2 & 34350 & 0.54 \\
\hline OZO863 & 71187 & 7.8 & Older Volcanics & -70.5 & 0.01 & 0.99 & -21.3 & 37040 & 0.46 \\
\hline OZO860 & 84032 & 14.5 & Older Volcanics & -54 & 0.01 & 6.27 & -16.3 & 22250 & -0.81 \\
\hline OZO857 & 107475 & 16.5 & Older Volcanics & -20 & 0.09 & 78.77 & -19.6 & 1915 & -0.96 \\
\hline
\end{tabular}

${ }^{\text {a }}$ ANSTO code

${ }^{\mathrm{b}}$ Identifier code for Victorian State Observation Bore Network

${ }^{\mathrm{c}}$ Minimum quantification limit $=0.15 \mathrm{TU}$ 